ARTICLE

\title{
A superconducting switch actuated by injection of high-energy electrons
}

\author{
M. F. Ritter ${ }^{1}$, A. Fuhrer (10 ${ }^{1 凶}$, D. Z. Haxell ${ }^{1}$, S. Hart ${ }^{2}$, P. Gumann ${ }^{2}$, H. Riel ${ }^{1} \&$ F. Nichele $\mathbb{B}^{1 凶}$
}

Recent experiments with metallic nanowires devices seem to indicate that superconductivity can be controlled by the application of electric fields. In such experiments, critical currents are tuned and eventually suppressed by relatively small voltages applied to nearby gate electrodes, at odds with current understanding of electrostatic screening in metals. We investigate the impact of gate voltages on superconductivity in similar metal nanowires. Varying materials and device geometries, we study the physical mechanism behind the quench of superconductivity. We demonstrate that the transition from superconducting to resistive state can be understood in detail by tunneling of high-energy electrons from the gate contact to the nanowire, resulting in quasiparticle generation and, at sufficiently large currents, heating. Onset of critical current suppression occurs below gate currents of 100fA, which are challenging to detect in typical experiments.

\footnotetext{
${ }^{1}$ IBM Research Europe, Rüschlikon, Switzerland. ${ }^{2}$ IBM T. J. Watson Research Center, Yorktown Heights, NY, USA. ${ }^{凶}$ email: afu@ibm.zurich.com; fni@ibm.zurich.com
} 
S uperconducting circuits, thanks to their ultra-low power consumption and high speed, offer great promise as building blocks for quantum computing architectures and related cryogenic control electronics. In this context, it is especially intriguing to develop switching devices that can be electrically tuned between a superconducting and a resistive state at high frequency. Ultimately, such a three-terminal device would enable novel functionalities for which no semiconducting counterpart exists, such as cryogenic switches, ultra-sensitive detectors, amplifiers, circulators, multiplexers, and frequency tunable resonators ${ }^{1-8}$. Several electrically controlled superconducting switches based on the injection of out-of-equilibrium quasiparticles in Josephson junctions have been realized ${ }^{9-12}$. However, Josephson junctions typically come with limited source-drain critical currents and the requirement to operate in magnetic field-free environments. Consequently, architectures that do not rely on Josephson junctions are subjected to intense study. Such pioneering approaches are based on three or four terminal devices where electrical currents ${ }^{13}$, locally generated Oersted fields ${ }^{14}$ or heat ${ }^{15-17}$ drive a superconducting channel normal. Finally, recent experiments suggest that moderate electric fields might affect superconductivity in metallic nanowires ${ }^{18,19}$. Controlling superconductivity in metallic devices via gate voltages would be appealing, however, a satisfactory explanation for the observed phenomena was not provided, yet.

Here, we report an experimental investigation of metallic nanowires subjected to electric fields. Our findings rule out any variation of superconducting properties as a direct consequence of the applied electric field, as suggested in refs. ${ }^{18,19}$. On the other hand, we highlight the importance of tunneling and field emission from the gate electrode. Detailed measurements indicate that relatively few electrons, injected at energies several orders of magnitude higher than the superconducting gap, trigger the generation of a large number of quasiparticles and weaken superconductivity. This is in stark contrast to previously demonstrated devices actuated by quasiparticle injection at low energy ${ }^{10,15,17}$, where gate currents comparable to the device critical current (a few $\mu \mathrm{A})$ were needed for switching. For larger gate currents, injected electrons locally increase temperature and drive the nanowires normal. We characterize the effect of electron injection into nanowires in terms of their critical current and its dependence on gate voltage, temperature, and magnetic field. This basic characterization is performed with different substrates and superconductors. We then investigate how injected quasiparticles influence superconductivity along the length of the channel in a region free of electric fields. After presenting the experimental observations, we will elaborate on their physical origins.

\section{Results}

Basic characterization. A typical device is shown in Fig. 1a together with its measurement setup: it consists of a $2 \mu \mathrm{m}$ long, $80 \mathrm{~nm}$ wide TiN wire (blue) with a TiN side gate (red). Wire and side gate are separated by an $80 \mathrm{~nm}$ wide gap. Gates and nanowires were defined by electron beam lithography and dry etching of a TiN film deposited on an intrinsic Si substrate (gray). Measurements were performed by low-frequency lock-in techniques by passing a source-drain current $I_{\mathrm{SD}}$ in the nanowire and recording the resulting voltage $V$. Gate voltage $V_{\mathrm{G}}$ was applied via a source-measure unit, which also recorded the current $I_{\mathrm{G}}$ entering the gate contact. A second side gate (gray) was not operated in this work and left grounded. Further details on materials, samples fabrication, and measurement techniques are reported in the Methods Section. At $V_{\mathrm{G}}=0$, the nanowire showed a critical current $I_{\mathrm{C}}=45 \mu \mathrm{A}$, measured sweeping $I_{\mathrm{SD}}$ in either direction starting in the superconducting state. In contrast, when sweeping $I_{\mathrm{SD}}$ from the normal state towards zero, superconductivity was re-established below the retrapping current $I_{\mathrm{R}}=1 \mu \mathrm{A}$. Figure $1 \mathrm{~b}$ shows the nanowire differential resistance $d V / d I_{\mathrm{SD}}$, measured while sweeping $I_{\mathrm{SD}}$ in the positive direction. The inset gives the temperature dependence of $I_{\mathrm{C}}$ and $I_{\mathrm{R}}$. The large difference between $I_{\mathrm{C}}$ and $I_{\mathrm{R}}$, especially marked at low temperature, is likely owing to self-heating when the nanowire is in the normal state, together with the difficulty in extracting heat via the substrate or the leads ${ }^{20}$. Figures $1 \mathrm{c}$, $\mathrm{d}$ show $I_{\mathrm{C}}$ and $I_{\mathrm{G}}$, respectively, as a function of $V_{\mathrm{G}}$. For $V_{\mathrm{G}} \sim \pm 2.5 \mathrm{~V}$, just before $I_{\mathrm{G}}$ reached detection level $\left(\sim 100 \mathrm{fA}\right.$ in our setup), $I_{\mathrm{C}}$ started

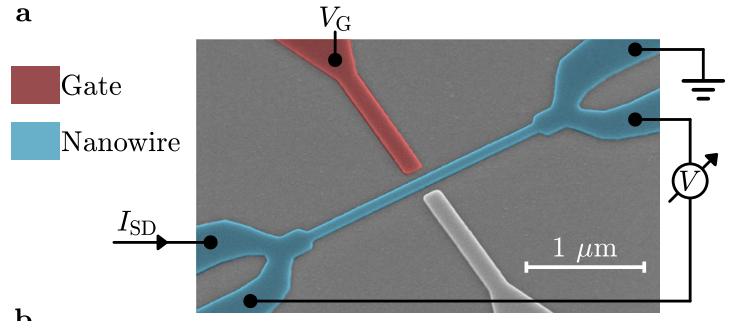

b

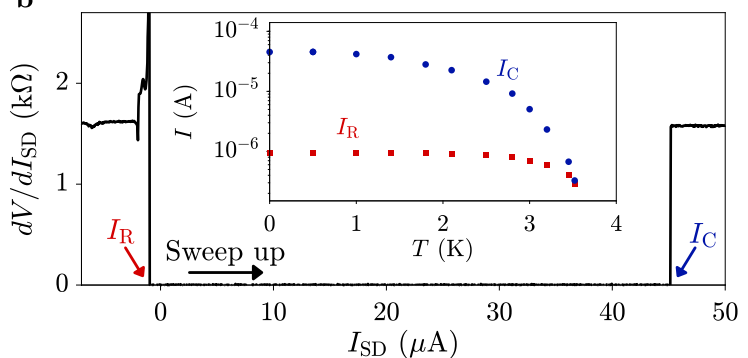

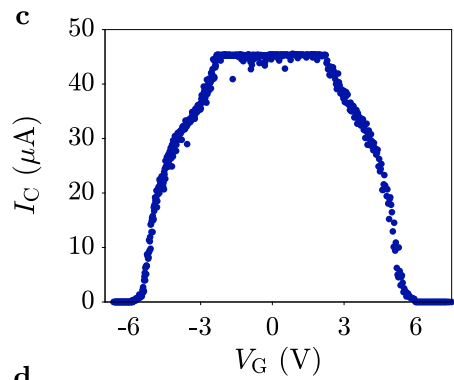

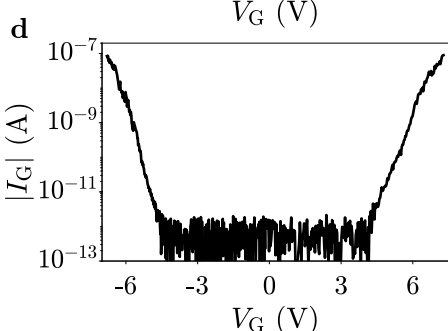



Fig. 1 Operation of a metallic nanowire superconducting switch. a False-color scanning electron micrograph of a device identical to that under study, together with a schematic of the measurement setup. The silicon substrate is gray, the TiN nanowire blue and the gate electrode red. Another gate electrode (gray) was left grouded. b Differential resistance $d V / d I_{S D}$ of the nanowire as a function of $I_{S D}$, measured by Sweeping up $I_{S D}$ starting from $-50 \mu \mathrm{A}$. Critical current $I_{C}$ and retrapping current $I_{R}$ are indicated. Inset: temperature dependence of $I_{C}$ (blue dots) and $I_{R}$ (red squares). c Critical current $I_{C}$ in the nanowire as a function of gate voltage $V_{\mathrm{G}}$. $\mathbf{d}$ Absolute value of the gate current $I_{\mathrm{G}}$ flowing between gate and nanowire as a function of $V_{\mathrm{G}}$. A linear component $I_{\mathrm{G}} \sim 1 \mathrm{~T} \Omega$, attributed to leakage in the measurement setup, was subtracted from the data (see Supplementary Note 1 ). e Parametric plot of $I_{C}$ vs. $I_{\mathrm{G}}$. Obtained from the data in $\mathbf{c}$ and $\mathbf{d}$. 

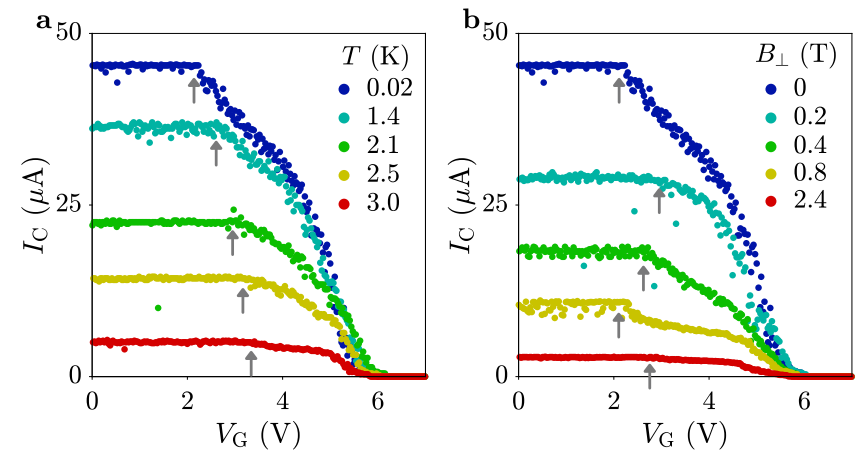

Fig. 2 Temperature and magnetic field dependence. a Critical current $I_{C}$ of the device presented in Fig. 1 as a function of gate voltage $V_{G}$ for various temperatures $T$ (see legend). $\mathbf{b}$ Critical current $I_{C}$ of the device presented in Fig. 1 as a function of out-of-plane magnetic fields $B_{\perp}$ (see legend). Gray arrows indicate the gate voltage where $I_{C}$ starts decreasing.
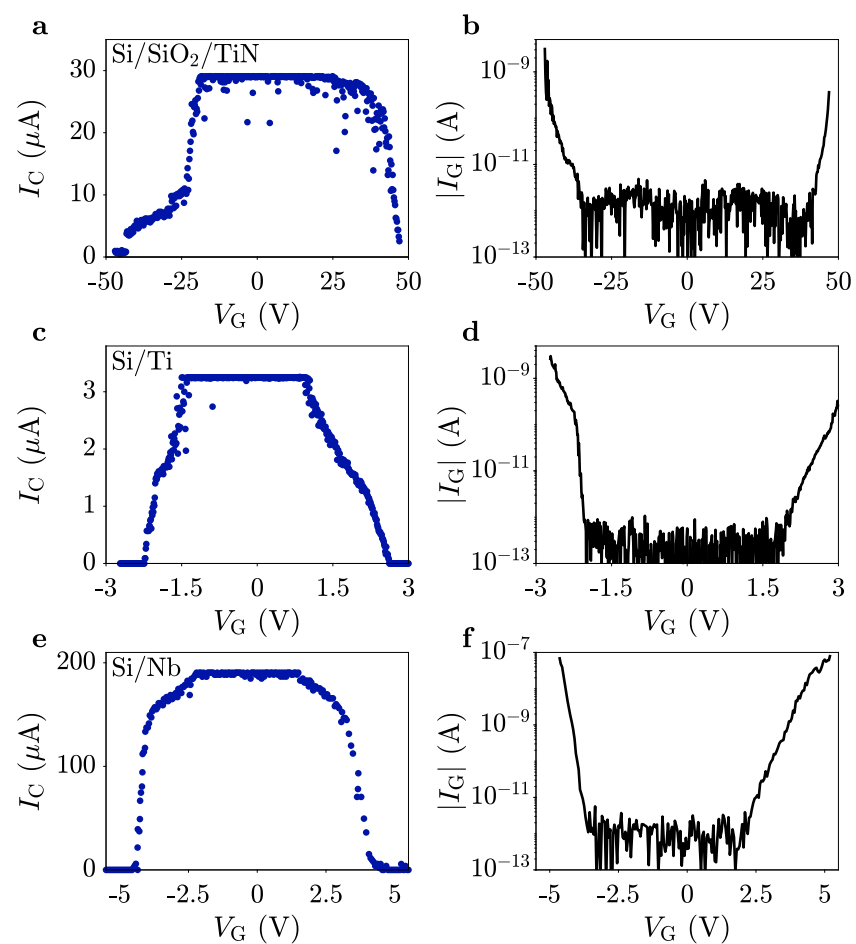

Fig. 3 Critical current suppression in various superconductors. $\mathbf{a}, \mathbf{b}$ Critical current $I_{C}$ and gate current $I_{G}$ as a function of gate voltage $V_{G}$ for a $\mathrm{TiN}$ wire on a $25 \mathrm{~nm}$ thick $\mathrm{SiO}_{2}$ film thermally grown on $\mathrm{Si}$ substrate. The wire is $2 \mu \mathrm{m}$ long, $80 \mathrm{~nm}$ wide, and $20 \mathrm{~nm}$ thick. c, d Critical current $I_{\mathrm{C}}$ and gate current $I_{\mathrm{G}}$ as a function of gate voltage $V_{\mathrm{G}}$ for a Ti wire on Si substrate. The wire is $2 \mu \mathrm{m}$ long, $200 \mathrm{~nm}$ wide, and $30 \mathrm{~nm}$ thick. e, f Critical current $I_{C}$ and gate current $I_{\mathrm{G}}$ as a function of gate voltage $V_{\mathrm{G}}$ for a $\mathrm{Nb}$ wire on $\mathrm{Si}$ substrate. The wire is $2 \mu \mathrm{m}$ long, $200 \mathrm{~nm}$, wide, and $13 \mathrm{~nm}$ thick.

decreasing. At $V_{\mathrm{G}}= \pm 5.5 \mathrm{~V}$, where $I_{\mathrm{G}} \approx \pm 1.5 \mathrm{nA}, I_{\mathrm{C}}$ vanished and the nanowire reached its normal state resistance $R_{\mathrm{N}}=1.6 \mathrm{k} \Omega$. The parametric plot of $I_{\mathrm{C}}$ vs. $I_{\mathrm{G}}$ shown in Fig. 1e indicates an extremely sharp suppression of $I_{\mathrm{C}}$ for small values of $I_{\mathrm{G}}(\sim 50 \%$ of the suppression took place within the noise level for $I_{\mathrm{G}}$ ), followed by a slower decay which persisted up to $I_{\mathrm{G}} \sim 1.5 \mathrm{nA}$.

Temperature and magnetic field dependence. Figure $2 \mathrm{a}$, b show the gate voltage dependence of $I_{C}$ for various temperatures $T$ and out-of-plane magnetic fields $B_{\perp}$, respectively. Neither temperature nor field affected the $I_{\mathrm{G}}$ vs. $V_{\mathrm{G}}$ characteristics of Fig. 1d (see Supplementary Note 1), and resulted in identical $V_{\mathrm{G}}$ values for complete suppression of superconductivity in the nanowires, up to the critical temperature and critical field. On the other hand, the increase of $I_{\mathrm{C}}$ suppression systematically moved to higher $V_{\mathrm{G}}$ for higher temperatures (see gray arrows). A more complicated dependence was observed as a function of $B_{\perp}$.

Critical current suppression in various superconductors. Suppression of $I_{\mathrm{C}}$ concomitant to, or slightly anticipating, the onset of $I_{\mathrm{G}}$ above detection level was confirmed for over $20 \mathrm{TiN}$ devices, characterized by various gate shapes, nanowire widths $(40,80$, and $200 \mathrm{~nm})$, nanowire lengths $(650 \mathrm{~nm}, 1$ and $2 \mu \mathrm{m})$, and gate-to-wire separations (80 and $160 \mathrm{~nm}$, see Supplementary Note 3). Similar behavior was also observed on devices with a different substrate than $\mathrm{Si}$ or with a different superconductor than TiN. Figures $3 \mathrm{a}, \mathrm{b}$ show measurements performed on a TiN device as that of Fig. 1a, but deposited on a $25 \mathrm{~nm} \mathrm{SiO}_{2}$ layer thermally grown on $\mathrm{Si}$. Despite the vastly different operational range of $V_{\mathrm{G}}$ with respect to that of Fig. 1, suppression of $I_{\mathrm{C}}$ still coincided with the onset of $I_{\mathrm{G}}$. Devices with a $\mathrm{SiO}_{2}$ interlayer further showed a characteristic asymmetry of the $I_{\mathrm{C}}$ vs. $V_{\mathrm{G}}$ curve, with a sharper suppression of $I_{\mathrm{C}}$ for negative than for positive $V_{\mathrm{G}}$. Given the sharp termination of the gate electrode, and the large electric field reached on $\mathrm{SiO}_{2}$ substrates, emission of electrons from the gate is expected to be easier for negative gate biases. In the present case, detection of small gate current asymmetries is hindered by spurious current leakage in the measurement setup for high gate biases. Figure $3 c, d$ show $I_{\mathrm{C}}$ and $I_{\mathrm{G}}$, respectively, as a function of $V_{\mathrm{G}}$ for a Ti nanowire as that of Fig. 1a, but with $200 \mathrm{~nm}$ width and $30 \mathrm{~nm}$ thickness. In this case, the normal state was reached for $I_{\mathrm{G}}$ as low as $30 \mathrm{pA}$ for positive $V_{\mathrm{G}}$. Figure $3 \mathrm{e}, \mathrm{f}$ show $I_{\mathrm{C}}$ and $I_{\mathrm{G}}$, respectively, as a function of $V_{\mathrm{G}}$ for a $\mathrm{Nb}$ nanowire as that of Fig. 1a but with $13 \mathrm{~nm}$ thickness. Similarly to the previous cases, $I_{\mathrm{C}}$ started decreasing with $I_{\mathrm{G}}$ still below $100 \mathrm{fA}$. However complete suppression of $I_{\mathrm{C}}$ required $I_{\mathrm{G}} \geq 20 \mathrm{nA}$. Overall, these results indicate that the switching mechanism presented here is generic, and not linked to specific superconductors or substrates. On the other hand, data also suggests that small gap superconductors (e.g., Ti) require considerably less gate current for switching to occur with respect to superconductors with larger gaps (e.g., TiN or $\mathrm{Nb}$ ).

Spatially resolved suppression of the critical current. Measurements presented so far were conducted in relatively short nanowires, where sharp transitions from zero resistance to the normal state were observed. We complement these observations with measurements on a long, multi-terminal nanowire, which allow us to investigate how superconductivity is affected away from the electron injection point, along the nanowire length. The device shown in Fig. 4a consists of six TiN segments of $1 \mu \mathrm{m}$ length and $80 \mathrm{~nm}$ width (named $A$ to $F$ ). Each segment $j$ is controlled by a nearby gate, with gate voltage $V_{\mathrm{G}}^{\mathrm{j}}$ and corresponding gate current $I_{\mathrm{G}}^{\mathrm{j}}$. In a first measurement configuration (Configuration 1), schematically shown in Fig. $4 \mathrm{~b}, I_{\mathrm{SD}}$ was passed between contacts 1 and 9, that is the DC current is the same for every segment. As $I_{\mathrm{SD}}$ was ramped, voltages $V_{j}$ across the six segments were simultaneously recorded. Critical currents $I_{\mathrm{C}}^{j}$, defined as the values of $I_{\mathrm{SD}}$ where segment $j$ turned resistive, are reported in Fig. $4 \mathrm{~b}$ as a function of $V_{\mathrm{G}}^{\mathrm{A}}$, with the corresponding gate current $I_{\mathrm{G}}^{\mathrm{j}}$ shown in Fig. $4 \mathrm{~d}$. Configuration 1 highlights two regimes. For $I_{\mathrm{SD}}>I_{\mathrm{R}}$, switching in all the segments happened simultaneously. For $I_{\mathrm{SD}}<I_{\mathrm{R}}$, switching was sequential: the further away a segment was from the biased gate, the larger was the gate current required to suppress its critical current. We contrast this 
a

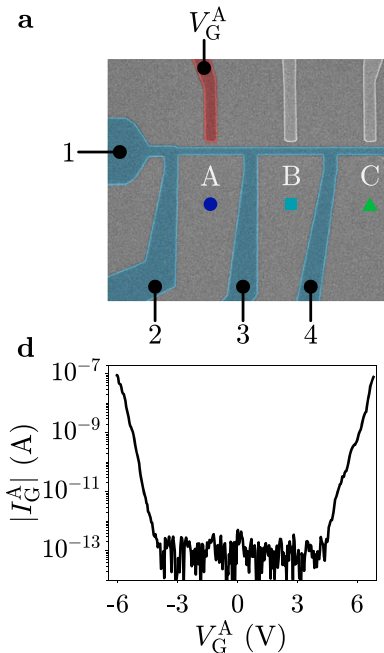

b

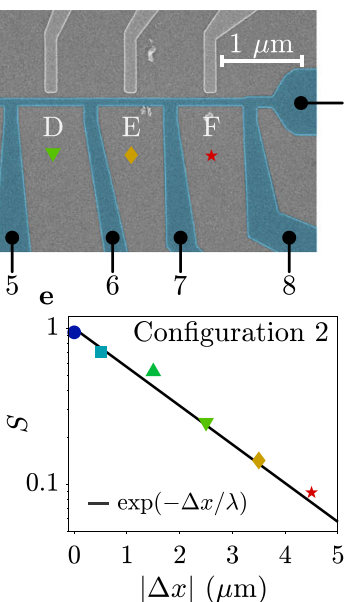

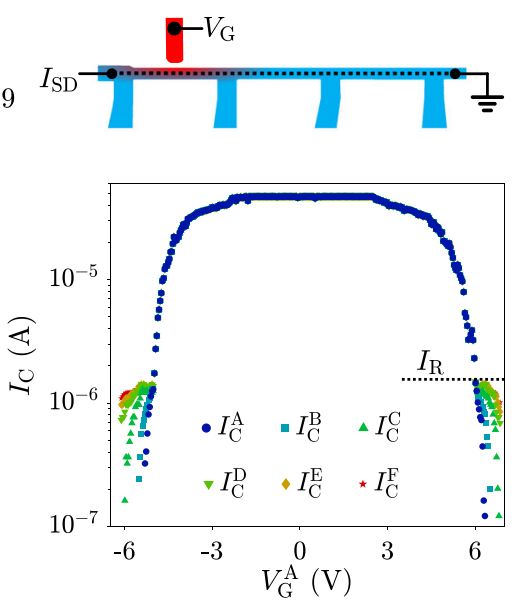

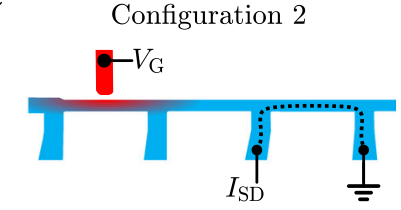

Fig. 4 Spatially resolved suppression of the critical current. a False-color scanning electron micrograph of a device as that under study. Colors are as in Fig. 1a. b Top: schematics of measurement configuration 1, where $I_{S D}$ always intersects the point of electron injection. Bottom: critical currents of the six segments as a function of gate voltage $V_{G}^{A}$. c Top: schematics of measurement configuration 2, where $I_{\mathrm{SD}}$ does not necessarily intersect the point of electron injection. Bottom: critical currents of the six segments as a function of gate voltage $V_{G}^{A}$. d Absolute value of gate current $I_{G}^{A}$ as a function of gate voltage $V_{G}^{A}$. e Suppression factor $S$ as a function of the distance between gate and nanowire segment, calculated from the data in $\mathbf{c}$ for $V_{G}^{A}=6 V_{\text {. The solid }}$ line is a fit to an exponential, resulting in a characteristic length scale $\lambda=1.8 \mu \mathrm{m}$.

behavior with the results obtained using Configuration 2, schematically shown in Fig. $4 c$. In this case, $I_{\mathrm{SD}}$ is routed in one segment only. The critical currents of the six segments were extracted in six separate measurements as $V_{\mathrm{G}}^{\mathrm{A}}$ was biased (see Fig. $4 \mathrm{c}$ ). Routing $I_{\mathrm{SD}}$ far from the electron injection point avoids the simultaneous switching observed in Fig. 4b, highlighting instead spatial dependence of the critical current also for $I_{\mathrm{SD}}>I_{\mathrm{R}}$. In Fig. 4e, we plot the critical current suppression factor $S$ as a function of distance $\Delta x$ between injection point and segment. The suppression factor for a segment $j$ is defined as $S^{j}=\left(I_{0}{ }^{j}-I_{C}{ }^{j}\right) / I_{0}{ }^{j}$, where $I_{0}{ }^{j}$ is the critical current of a segment for zero gate voltage. A fit to an exponentially decaying function $\exp (-\Delta x / \lambda)$ (solid line in Fig. 4f), yields a characteristic decay length $\lambda \sim 1.8 \mu \mathrm{m}$.

\section{Discussion}

After presenting the experimental results, we now discuss the origin of the observed phenomena. Injected electrons reach the superconductor in a deeply out-of-equilibrium state, with energies of the order $e V_{\mathrm{G}}$, much larger than the superconducting gap $\Delta\left(\Delta=500 \mu \mathrm{eV}\right.$ for $\left.\mathrm{TiN}^{21}\right)$. As each electron relaxes to the gap edge by inelastic scattering with other electrons and phonons, up to $e V_{\mathrm{G}} / \Delta \sim 10^{5}$ quasiparticles are generated within the nanowire. A sufficiently high concentration of quasiparticles drives the nanowire normal by quenching the superconducting gap ${ }^{22}$ and suppression of the depairing critical current ${ }^{23}$, leading to a switch to the normal state. The closer $I_{\mathrm{SD}}$ is to $I_{\mathrm{C}}$, the more sensitive the device becomes, so that relatively few injected electrons can trigger a normal state transition. Indeed, half of the suppression of $I_{\mathrm{C}}$ takes place for gate currents below the noise floor of our setup, where the power provided by the gate voltage source is less than $300 \mathrm{fW}$ and unlikely to result in any relevant temperature increase. Indeed, a temperature increase $\sim 1.5 \mathrm{~K}$ would be needed for an appreciable variation of $I_{C}$ to be detected (see Fig. 1b). This can be is excluded at such small gate currents. Such behavior is reminiscent of superconducting nanowire single-photon detectors (SNSPDs) ${ }^{24,25}$, where the strike of a visible or infrared photon promotes a single electron to high energy, which in turn triggers the generation of a large amount of quasiparticles as it relaxes. In the present case, high-energy charge carriers are provided directly by the gate current. Owing to their close proximity, gate, and nanowire are coupled by phonons, so that dissipation of the injected energy and generation of quasiparticles occurs on both sides. Figures $2 \mathrm{a}, \mathrm{b}$ show rich physics at low values of $V_{\mathrm{G}}$, with the initial suppression of $I_{\mathrm{C}}$ moving to higher and higher gate voltages as temperature increases (see gray arrows). This behavior presumably reflects the increase of quasiparticle density in the wire with temperature, requiring more electrons to be injected before a sizable effect on $I_{\mathrm{C}}$ is observed. Systematic studies of the more complicated variations of $I_{\mathrm{C}}$ vs. $V_{\mathrm{G}}$ as a function of $B_{\perp}$ could shed new light on the physics of field repulsion and vortex penetration in nanowires ${ }^{26}$.

For gate currents several orders of magnitude larger, the power provided to the device is significant and likely to result in an increase of the local lattice temperature. We estimate the minimum power required for keeping the nanowire in the normal state as $P_{\mathrm{R}}=I_{\mathrm{R}}{ }^{2} R_{\mathrm{N}}$, which is Joule heating in the normal state and at the retrapping current. For the device of Fig. 1 we obtain $P_{\mathrm{R}}=1.6 \mathrm{nW}$. This power is similar to one provided by the gate voltage source at the point where superconductivity is suppressed $P_{\mathrm{G}}=V_{\mathrm{G}} I_{\mathrm{G}}$. For the device of Fig. 1, we obtain $P_{\mathrm{G}}=6.1 \mathrm{nW}$. The difference between $P_{\mathrm{R}}$ and $P_{\mathrm{G}}$ is readily accounted for by considering that a significant fraction of $P_{\mathrm{G}}$ is not dissipated in the nanowire but in the gate electrode and in the surrounding environment. Furthermore, quasiparticles generated within the nanowire spread over a distance longer than the nanowire length, so that a fraction of them thermalizes in the leads (see the following discussion). The relation $P_{\mathrm{G}} \approx 4 P_{\mathrm{R}}$ is closely followed also for the devices of Fig. 3. The consistency is remarkable considering that dissipated power in the Nb wire $\left(P_{\mathrm{R}}=29 \mathrm{nW}\right)$ is three orders of magnitude larger than for the Ti wire $\left(P_{\mathrm{R}}=23 \mathrm{pW}\right)$.

After determining that small currents of high-energy electrons are responsible for weakening the superconducting properties, we discuss in more detail how the transition to the normal state takes place. The device of Fig. 1 showed a sharp transition from superconducting to its normal state resistance for any gate voltage. This behavior might appear surprising considering that the gate acts on a short portion of the nanowire. With reference to Fig. 4, we demonstrated that the sharp transition to the normal state resistance is a result of the measurement configuration. 
Indeed, $I_{\mathrm{C}}$ is first reduced in a region close to the point of electron injection. As $I_{\mathrm{SD}}$ is increased, that region switches to the resistive state and becomes a hotspot due to the large $I_{\mathrm{SD}}$ flowing in the nanowire. For $I_{\mathrm{SD}}>I_{\mathrm{R}}$ the hotspot warms up the surrounding metal via Joule heating, resulting in a further spreading of the normal region. This process rapidly turns normal the entire nanowire length along the path of $I_{\mathrm{SD}}$. For $I_{\mathrm{SD}}<I_{\mathrm{R}}$ the power dissipated in the hotspot is insufficient to trigger the transition to the normal state in the nearby metal. In this case, considerable gate currents are needed for influencing the regions of the nanowire, which are furthest away via diffusion of energetic quasiparticles and heat. In Configuration 2, $I_{\mathrm{SD}}$ does not intersect the point of electron injection (except for segment A) and simultaneous switching is prevented and the critical current is lowest at the point of injection and restored at large distances. The characteristic length scale of $1.8 \mu \mathrm{m}$ is presumably related to the diffusion length of long-lived quasiparticles. A framework for calculating quasiparticle density profiles has been put forward for SNSPDs in ref. ${ }^{26}$ and is compatible with our experimental results.

Recent work argued on the effect of electric fields on the critical current of metallic nanowires, using a similar device as that of Fig. $1 \mathrm{a}^{18,19}$. From a qualitative standpoint, the modulation of $I_{\mathrm{C}}$ we observe strongly resembles data in refs. ${ }^{18,19}$, including ambipolar behavior, response to temperature and magnetic field, and spacial suppression of the supercurrent reduction. We note that electric field modulation of superconductivity has been previously demonstrated in metallic thin films ${ }^{27-29}$, but changes of critical temperature by less than one percent required significantly stronger electric fields than those applied here. Investigating multiple material combinations and comparing local and non-local measurement configurations allow us to readily exclude any electric field-induced suppression of superconductivity in our devices. First, gate voltages are too small to generate a sizable electric field (see Supplementary Note 3 for a different gate design leading to identical results). Second, suppression of superconductivity is always correlated to the onset of gate currents, irrespective of the applied gate voltage. Third, non-local responses extend far beyond the gate induced electric field, to a distance where only quasiparticle diffusion and phonons are relevant (see Fig. 4). We further note that the gate current needed for affecting superconductivity is especially low for small gap superconductors on highly insulating substrates, the platform used in refs. ${ }^{18,19}$. For large gap superconductors on semi-insulating substrates, i.e., the main focus of this work, the lower energy of emitted electrons, together with the requirement for higher quasiparticle density to suppress superconductivity, make required gate currents larger and their detection more feasible.

Injection of high-energy electrons in superconducting elements might be employed for the realization of fast and electrically controlled superconducting switches. As devices properly function also in the limit of $I_{\mathrm{SD}}=0$, self-resetting from normal to superconducting state is not inhibited by self-heating ${ }^{17}$, a limitation of other superconducting devices. The quasiparticle relaxation length limits the extent of the segment that can be switched, and consequently the largest normal state resistance of the device. Alternatively, nanowires of arbitrary length can be operated by choosing $I_{\mathrm{SD}}>I_{\mathrm{R}}{ }^{13,15}$. We expect the switching time to be fast, presumably limited by quasiparticle recombination $(<100 \mathrm{ps})^{15}$. In our case, we measured a switching time below 100 ns, limited by the setup in use (see Supplementary Note 4).

In conclusion, we demonstrated quenching of superconductivity in metallic nanowires via gate currents several orders of magnitude smaller than the source-drain critical current and described the physical mechanisms responsible for this behavior. Devices studied here could serve as tools for novel studies of quasiparticle physics at unprecedented energy scales and in the limit of no current flowing in the nanowire. Furthermore, combining length dependence studies as in Fig. 4 with time-resolved measurements, will provide a novel tool to investigate quasiparticle dynamics and thermal effects.

\section{Methods}

Sample fabrication. Devices were obtained on either intrinsic Si substrates or intrinsic $\mathrm{Si}$ substrates with a $25 \mathrm{~nm}$ thermally grown $\mathrm{SiO}_{2}$ top layer. Both high and low resistivity $\mathrm{Si}$ resulted in similar device performance at low temperature in terms of gate currents vs. gate voltages. Just prior to the deposition of the superconductor, Si chips were immersed in a buffered HF solution for removing the native Si oxide. Nanowires were defined by electron beam lithography, as detailed below. After the nanowires were fabricated, Ti/Au bonding pads, placed $\sim 170 \mu \mathrm{m}$ away from the active region of the devices, were defined by optical lithography, thermal evaporation, and lift-off.

TiN wires were obtained from a $20 \mathrm{~nm}$ TiN film was deposited by DC reactive magnetron sputtering. A $50 \mathrm{~nm}$ thick layer of hydrogen silsesquioxane (HSQ) based negative tone resist was used as mask. After resist development, unprotected $\mathrm{TiN}$ areas were removed by inductively coupled plasma etching in $\mathrm{HBr}$ plasma. After etching of the TiN, HSQ was removed by immersion in a diluted hydrofluoric acid solution. Characterization of the TiN film gave a resistivity of $68 \Omega$ per square, a critical temperature of $3.7 \mathrm{~K}$ and a critical out-of-plane magnetic field of $3.5 \mathrm{~T}$. These properties remained unchanged in the completed devices.

Ti wires were defined by electron beam lithography on a positive tone polymethyl methacrylate mask, electron beam evaporation of a $30 \mathrm{~nm}$ thick Ti film and lift-off. Ti evaporation was performed at a base pressure of $10^{-9} \mathrm{mbar}$ and at a deposition rate of $1 \mathrm{~nm} \mathrm{~s}^{-1}$. The high deposition rate was chosen to minimize contamination of the Ti film during evaporation ${ }^{30}$. The Ti wire of Fig. $3 \mathrm{c}$ had a critical temperature of $220 \mathrm{mK}$, a normal state resistance of $74 \Omega$ and a retrapping current of $560 \mathrm{nA}$.

$\mathrm{Nb}$ wires were obtained by sputtering of a $13.5 \mathrm{~nm}$ film on intrinsic Si substrates and following a fabrication similar to that described above for TiN. Dry etching was performed with $\mathrm{Ar} / \mathrm{Cl}_{2}$ plasma. The $\mathrm{Nb}$ wire of Fig. $3 \mathrm{e}$ had a normal state resistance of $655 \Omega$ and a retrapping current of $7.6 \mu \mathrm{A}$.

Electrical measurements. Unless differently specified, measurements were performed in a dilution refrigerator at the base temperature of $10 \mathrm{mK}$. Low-pass RC filters and microwave pi-filters were installed along each line. A DC source-drain current $I_{\mathrm{SD}}$, superimposed to a small AC component of $30 \mathrm{nA}$ and $113 \mathrm{~Hz}$ was applied to the nanowire via large bias resistors. The AC differential voltage $V$ across the nanowire was then recorded with lock-in amplifiers with $10 \mathrm{M} \Omega$ input impedance and used to calculate the differential resistance $d V / d I_{\mathrm{SD}}$. Measurements were recorded with $I_{\mathrm{SD}}$ as the fast axis, sweeping from zero to positive values. This allowed initializing the wires to the superconducting state before each sweep started. Gate voltages were applied with a Keysight B2902A source-measure unit, which also recorded the current entering the gate contact. To avoid damaging the devices, a compliance of $\pm 95 \mathrm{nA}$ was chosen. A linear contribution of $\sim 1 \mathrm{pAV}^{-1}$, associated with spurious leakage paths in our setup, was subtracted from the $I_{\mathrm{G}}$ measurements shown in the Main Text (see Supplementary Note 1). To avoid potential contributions from displacement currents or hysteresis, $I_{\mathrm{G}}$ values were recorded by sweeping $V_{\mathrm{G}}$ from $0 \mathrm{~V}$ towards either positive or negative voltages and waiting times in excess of $30 \mathrm{~s}$ were allowed. Plots as that of Fig. 1c, d were then obtained by merging two data sets at $V_{\mathrm{G}}=0 \mathrm{~V}$. In case a second gate was present and left grounded, as for the device in Fig. 1a, it was verified that most of the gate current was flowing from the energized gate to the nanowire and not to the grounded gate.

In a standard measurement configuration, $I_{\mathrm{SD}}$ is sourced on one side of the sample by applying a voltage to a large resistor, whereas the other side of the sample is connected to ground. This asymmetric configuration results in lifting of the nanowire potential by $I_{\mathrm{SD}} R_{\mathrm{L}}$, where $R_{\mathrm{L}}$ is the resistance of the line connecting the nanowire to ground $(2.2 \mathrm{k} \Omega$ in our case). This effect is typically negligible, except for samples where a large $I_{\mathrm{SD}}$ is required (see Fig. 3e). A first approach is to use a symmetric current biasing configuration, as shown in Supplementary Fig. 4. However, using large resistors on both ends of the nanowire strongly reduces the available bandwidth. Alternatively, this effect can be accounted for by representing critical currents on the virtual voltage axis $V_{\mathrm{G}}{ }^{*}=V_{\mathrm{G}}-I_{\mathrm{SD}} R_{\mathrm{L}}$.

\section{Data availability}

The data that support the findings of this study are available from the corresponding authors on reasonable request.

Received: 16 August 2020; Accepted: 29 December 2020; Published online: 24 February 2021

\section{References}

1. Faris, S. M., Raider, S. I., Gallagher, W. J. \& Drake, R. E. Quiteron. IEEE Trans. Magn. 19, 1293 (1983). 
2. Pepe, G. P. et al. Superconducting device with transistor-like properties including large current amplification. Appl. Phys. Lett. 77, 447 (2000).

3. Day, P. K., Leduc, H. G., Mazin, B. A., Vayonakis, A. \& Zmuidzinas, J. A broadband superconducting detector suitable for use in large arrays. Nature 425, 814 (2003).

4. Lee, S.-B., Hutchinson, G. D., Williams, D. A., Hasko, D. G. \& Ahmed, H. Superconducting nanotransistor based digital logic gates. Nanotechnology 14, 188-191 (2003).

5. Leduc, H. G. et al. Titanium nitride films for ultrasensitive microresonator detectors. Appl. Phys. Lett. 97, 102509 (2010).

6. Ho Eom, B., Day, P. K., LeDuc, H. G. \& Zmuidzinas, J. A wideband, low-noise superconducting amplifier with high dynamic range. Nat. Phys. 8, 623 (2012).

7. Hornibrook, J. M. et al. Cryogenic control architecture for large-scale quantum computing. Phys. Rev. Appl. 3, 024010 (2015).

8. Gasparinetti, S. et al. Fast electron thermometry for ultrasensitive calorimetric detection. Phys. Rev. Appl. 3, 014007 (2015).

9. Wong, T.-w, Yeh, J. T. C. \& Langenberg, D. N. Quasiparticle-injectioninduced superconducting weak links. Phys. Rev. Lett. 37, 150-153 (1976).

10. Morpurgo, A. F., Klapwijk, T. M. \& van Wees, B. J. Hot electron tunable supercurrent. Appl. Phys. Lett. 72, 966-968 (1998).

11. Giazotto, F. \& Pekola, J. P. Josephson tunnel junction controlled by quasiparticle injection. J. Appl. Phys. 97, 023908 (2005).

12. Nevirkovets, I. P. A superconducting transistorlike device having good inputoutput isolation. Appl. Phys. Lett. 95, 052505 (2009).

13. Wagner, A., Ranzani, L., Ribeill, G. \& Ohki, T. A. Demonstration of a superconducting nanowire microwave switch. Appl. Phys. Lett. 115, 172602 (2020).

14. Buck, D. A. The cryotron - a superconductive computer component. Proc. IRE 44, 482 (1956).

15. McCaughan, A. N. \& Berggren, K. K. A superconducting-nanowire threeterminal electrothermal device. Nano Lett. 14, 5748-5753 (2014).

16. Toomey, E. et al. Bridging the gap between nanowires and josephson junctions: a superconducting device based on controlled fluxon transfer. Phys. Rev. Appl. 11, 034006 (2019).

17. McCaughan, A. N. et al. A superconducting thermal switch with ultrahigh impedance for interfacing superconductors to semiconductors. Nat. Electron. 2, 451-456 (2019).

18. De Simoni, G., Paolucci, F., Solinas, P., Strambini, E. \& Giazotto, F. Metallic supercurrent field-effect transistor. Nat. Nanotechnol. 13, 802-805 (2018).

19. Paolucci, F. et al. Field-effect control of metallic superconducting systems. AVS Quantum Sci. 1, 016501 (2019).

20. Li, P. et al. Retrapping current, self-heating, and hysteretic current-voltage characteristics in ultranarrow superconducting aluminum nanowires. Phys. Rev. B 84, 184508 (2011).

21. Pracht, U. S. et al. Direct observation of the superconducting gap in a thin film of titanium nitride using terahertz spectroscopy. Phys. Rev. B 86, 184503 (2012).

22. Semenov, A. D., Gol'tsman, G. N. \& Korneev, A. A. Quantum detection by current carrying superconducting films. Phys. C 351, 349 (2001).

23. Semenov, A., Engel, A., Hübers, H.-W., Il'in, K. \& Siegel, M. Spectral cut-off in the efficiency of the resistive state formation caused by absorption of a singlephoton in current-carrying superconducting nano-strips. Eur. Phys. J. B 47, 495-501 (2005).

24. Kadin, A. M. \& Johnson, M. W. Nonequilibrium photon-induced hotspot: a new mechanism for photodetection in ultrathin metallic films. Appl. Phys. Lett. 69, 3938-3940 (1996).

25. Gol'tsman, G. N. et al. Picosecond superconducting single-photon optical detector. Appl. Phys. Lett. 79, 705-707 (2001).

26. Engel, A. \& Schilling, A. Numerical analysis of detection-mechanism models of superconducting nanowire single-photon detector. J. Appl. Phys. 114, 214501 (2013)
27. Glover, R. E. \& Sherrill, M. D. Changes in superconducting critical temperature produced by electrostatic charging. Phys. Rev. Lett. 5, 248-250 (1960).

28. Choi, J. et al. Electrical modulation of superconducting critical temperature in liquid-gated thin niobium films. Appl. Phys. Lett. 105, 012601 (2014).

29. Piatti, E. et al. Control of bulk superconductivity in a bcs superconductor by surface charge doping via electrochemical gating. Phys. Rev. B 95, 140501 (2017).

30. Wang, Z. et al. Electron-beam evaporated superconducting titanium thin films for antenna-coupled transition edge sensors. IEEE Trans. Appl. Supercond. 28, $1-4$ (2018).

\section{Acknowledgements}

We thank A. Pushp, B. Madon, and M.A. Mueed for deposition of the TiN films. We thank G. Salis, W. Riess, S. Olivadese, and J. Bulzacchelli for fruitful discussions, A. Olziersky, S. Paredes, and U. Drechsler for technical help. F. Nichele acknowledges support from the European Research Commission, grant no. 804273.

\section{Author contributions}

F.N. conceived the experiments. A.F. and F.N. designed the samples. S.H and P.G. deposited the Ti and $\mathrm{Nb}$ films. M.F.R. and D.Z.H. fabricated the devices. M.F.R., A.F., D.Z.H., and F.N. performed the measurements. M.F.R., A.F., D.Z.H, H.R., and F.N. interpreted and analyzed the data. A.F. and F.N. wrote the manuscript.

\section{Competing interests}

The authors declare no competing interests.

\section{Additional information}

Supplementary information The online version contains supplementary material available at https://doi.org/10.1038/s41467-021-21231-2.

Correspondence and requests for materials should be addressed to A.F. or F.N.

Peer review information Nature Communications thanks Alfredo Levy Yeyati and the other, anonymous, reviewer(s) for their contribution to the peer review of this work.

Reprints and permission information is available at http://www.nature.com/reprints

Publisher's note Springer Nature remains neutral with regard to jurisdictional claims in published maps and institutional affiliations.

Open Access This article is licensed under a Creative Commons Attribution 4.0 International License, which permits use, sharing, adaptation, distribution and reproduction in any medium or format, as long as you give appropriate credit to the original author(s) and the source, provide a link to the Creative Commons license, and indicate if changes were made. The images or other third party material in this article are included in the article's Creative Commons license, unless indicated otherwise in a credit line to the material. If material is not included in the article's Creative Commons license and your intended use is not permitted by statutory regulation or exceeds the permitted use, you will need to obtain permission directly from the copyright holder. To view a copy of this license, visit http://creativecommons.org/ licenses/by/4.0/

(C) The Author(s) 2021 